\title{
Outbreak and impact of coronavirus disease 2019 (COVID-19) on dialysis patients; Al Khezam dialysis center experience, Kuwait
}

\author{
Emad Abdallah $^{*(}$, Bassam Al Helal, Reem Asad, George Nessim, Shaikha Al-Bader, Yasser Elsharkawi, Ahmed \\ Elmasry, Mohamed Abdelgelil, Essam Allam, Sahar Abdelkareem, Mohammad Kamal, Mohamed Hemida, Yasser \\ Shaaban, Zeinab Zeid, Amr Saad, Abuhashem Ahmed
}

\begin{abstract}
Introduction: Coronavirus disease 2019 (COVID-19) is an outbreak due to SARS-CoV-2, declared by the World Health Organization (WHO) as a global pandemic in March 2020. Patients with underlying diseases, such as those with end-stage kidney disease (ESKD) on dialysis, are at greater risk.

Objectives: The aim of our study to assess the outbreak and impact of COVID-19 on dialysis patients.

Patients and Methods: Our study prospectively assessed and followed 442 patients with ESKD undergoing dialysis [390 patients on maintenance hemodialysis (HD) and 52 patients on peritoneal dialysis (PD)] for outbreak and impact of COVID-19 on these patients during the period from April 22, 2020 until March 23, 2021 in Al Khezam dialysis center, Kuwait. Age, gender, nationality, original kidney disease, history of hypertension (HTN), diabetes mellitus (DM), ischemic heart disease (IHD), congestive heart failure (CHF), bronchial asthma (BA), chronic obstructive pulmonary disease (COPD), history of pulmonary embolism (PE) and source of infection were analyzed. Symptoms as fever, fatigue, cough, loss of smell and taste and chest pain were recorded, the need for ICU admission, mechanical ventilation (MV), extracorporeal membrane oxygenation (ECMO), medications were recorded. The need to shift to continuous renal replacement therapy (CRRT) and outcomes (complications and mortality) were analyzed.

Results: Our study reported that 102 out of 442 (23\%) dialysis patients [97 out of $390(24.8 \%)$ HD patients and 5 out of 52 (9.6\%) PD patients] got infected with COVID-19 and reinfection reported in 4 out of 97 (4\%) COVID-19 HD patients. Around 27\% of COVID-19 HD patients had fever, $19 \%$ had fatigue, $8 \%$ had cough, $4 \%$ had loss of smell, $4 \%$ had loss of taste, $4 \%$ had chest pain and $40 \%$ of COVID-19 PD patients had fever. Fifteen out of 97 (15\%) COVID-19 HD patients needed ICU admission, 12 out of 97 (12\%) COVID-19 HD patients needed MV. A 33 out of 97 (34\%) COVID-19 HD patients and 4 out of 5 (80\%) COVID-19 PD patients needed to switch to CRRT. Mortality was 17 (15 HD and 2 PD) out of 102 (16.6\%) COVID-19 dialysis patients and common causes of death were sepsis, myocardial infarction (MI), heart failure and PE.

Conclusion: Outbreak and mortality of COVID-19 infection is high in ESKD patients undergoing dialysis compared with general populations. Strict protocol for prevention of COVID-19 should be undertaken in dialysis centers and encourage of home dialysis and highly protective COVID-19 vaccination priority for dialysis patients.

Keywords: COVID-19, Hemodialysis, Peritoneal dialysis

Citation: Abdallah E, Al Helal B, Asad R, Nessim G, Al-Bader SH, Elsharkawi Y, Elmasry A, Abdelgelil M, Allam E, Abdelkareem S, Kamal M, Hemida M, Shaaban Y, Zeid Z, Saad A, Ahmed A. Outbreak and impact of coronavirus disease 2019 (COVID-19) on dialysis patients; Al Khezam dialysis center experience, Kuwait. J Renal Endocrinol. 2021;7:e14. doi: 10.34172/jre.2021.14.

Copyright (C) 2021 The Author(s); Published by Nickan Research Institute. This is an open-access article distributed under the terms of the Creative Commons Attribution License (http://creativecommons.org/licenses/by/4.0), which permits unrestricted use, distribution, and reproduction in any medium, provided the original work is properly cited.
\end{abstract}

\section{Introduction}

Coronavirus disease 2019 (COVID-19) is an outbreak due to severe acute respiratory syndrome coronavirus 2 (SARS-CoV-2), a new virus of Coronaviridae family, emerged in China in December 2019 and declared by the World Health Organization as a global pandemic in March 2020. COVID-19 infection causes an atypical flu that often leads to severe acute respiratory syndrome with high mortality rate. Patients with underlying diseases, such as those with end-stage kidney disease (ESKD) on dialysis, are at greater risk (1).
In particular, COVID-19 is a real challenge for patients on HD, whose high susceptibility to COVID-19 is only partially explained by their average old age, frequent comorbidities, and impaired immune function. In fact, those receiving maintenance hemodialysis (HD). These patients cannot be isolated at home completely because they require routine treatment in the HD center, usually 3 times a week, the need of physical presence at health care facilities, the mobility to HD centers, and the high aggregation for the continuous physical proximity of patients during HD session strongly increase the risk of 
Implication for health policy/practice/research/ medical education

Our study prospectively assessed and followed 442 patients with endstage kidney disease (ESKD) undergoing dialysis $[390$ patients on maintenance hemodialysis (HD) and 52 patients on peritoneal dialysis (PD)] for outbreak and impact of COVID-19 on these patients during the period from April 22, 2020 until March 23, 2021 in Al Khezam dialysis center, Kuwait. Our study reported that $23 \%$ of dialysis patients $[24.8 \%$ HD patients and $9.6 \%$ PD patients] got infected with COVID-19 and reinfection reported in $4 \%$ of COVID-19 HD patients. Mortality was $16.6 \%$ of COVID-19 dialysis patients and common causes of death were sepsis, myocardial infarction, heart failure and pulmonary embolism.

contamination with SARS-CoV-2, the virus that causes COVID-19 (2). Surprisingly, first available data suggest that although HD patients' infection rate in the COVID-19 epidemic is much higher than in other populations, the disease course is rarely fatal and less severe, both compared with kidney-transplanted patients and general population (2).

\section{Objectives}

The aim of our study is to assess outbreak and impact of COVID-19 on dialysis patients in Al Khezam dialysis center, Kuwait during the period from April 22, 2020 until March 23, 2021.

\section{Patients and Methods}

\section{Study design}

Our study prospectively assessed and followed 442 patients with ESKD undergoing dialysis [390 patients on maintenance $\mathrm{HD}$ and 52 patients on peritoneal dialysis (PD)] for outbreak and impact of COVID-19 on these patients during the period from April 22, 2020 until March 23, 2021. Age, gender, nationality, original kidney disease, history of hypertension (HTN), diabetes mellitus (DM), ischemic heart disease (IHD), congestive heart failure (CHF), bronchial asthma (BA), chronic obstructive pulmonary disease (COPD), history of deep venous thrombosis (DVT) and pulmonary embolism (PE) and source of infection were analyzed. Symptoms as fever, fatigue, and cough, loss of smell and taste and chest pain were recorded. The need for intensive care unit (ICU) admission, mechanical ventilation (MV), extracorporeal membrane oxygenation (ECMO), inotropes, and medications were recorded. Angiotensinconverting enzyme inhibitor (ACEI)/angiotensin receptor blockers (ARBs) use prior to COVID-19 and ACEI/ ARBs held were analyzed. The need to shift to continuous renal replacement therapy (CRRT) and outcomes (complications and mortality) were also analyzed.

\section{Protocol for prevention of COVID-19 in Al Khezam dialysis center}

1. HD patients confirmed to have COVID-19 were transferred to the HD center specifically set up for confirmed COVID-19 cases in Jaber hospital, Kuwait for conducting dialysis.

2. HD patients in the primary HD center who had made contact with the patient were listed as close contacts and doing dialysis in isolation rooms for 14 days.

3. HD patients in the primary HD center who were on the same shift of dialysis remained in their initial hospital for dialysis and arrangements were made for them to receive dialysis in the same shift.

4. Patients were asked to isolate at home during the nondialysis period, and they were not allowed to take public transport to the hospital on the day of dialysis.

5. After patients arrived at the hospital, their body temperature and the epidemiological form (including whether there are any COVID-19 symptoms, suspected contact history and recent travel history) were recorded and verified by the physician.

6. HD patients under quarantine (history of contact to COVID-19 positive or recent returning from travel) continue their dialysis sessions during quarantine period at their usual center in isolation rooms.

7. Until the previous shift of dialysis patients left completely, the patients could not enter the dialysis treatment room.

8. PD patients should stay at home and hospital visits should be minimized for only urgent indications (e.g. suspected peritonitis).

9. PD patients with mild or moderate COVID-19 disease can continue PD treatment as usual and isolation at home.

10. PD patients with severe or critical cases can be temporarily transferred to $\mathrm{HD}$ and isolated in the hospital.

11. Medical masks were required for all patients during the entire dialysis treatment.

12. At the same time, the medical staff implemented strict hand hygiene policies and all members were required to use protective equipment, including surgical masks, face shields/goggles, protective gowns, medical caps, and gloves (3).

\section{Ethical issues}

The research followed the tenets of the Declaration of Helsinki written and informed consent taken from all participants before any intervention.

\section{Statistical analysis}

The mean and standard deviation and percentage of the results and paired $t$ tests to compare outbreak and mortality in diabetic, hypertensive, IHD and CHF patients with outbreak and mortality in patients with none DM, HTN, IHD and CHF were conducted. All statistical analyses were performed using MedCalc software (version 5, 2019 MedCalc, San Diego, CA, USA). $P$ value of 0.05 was used as a significance level. 


\section{Results}

Our study reported that 102 0ut of 442 (23\%) dialysis patients [97 out of $390(24.8 \%)$ HD patients and 5 out of 52 (9.6\%) PD patients] got infected with COVID-19 and reinfection reported in 4 out of 97 (4\%) COVID-19 HD patients during the period from April 22, 2020 until March 23, 2021 compared with 5.1\% in general population in Kuwait (Figure 1). Mean age, gender, nationality, original kidney disease, history of HTN, DM, IHD, CHF, BA, COPD, history of DVT and PE, source of infection and medications are shown in Table 1 . Around $27 \%$ of COVID-19 HD patients had fever, $19 \%$ had fatigue, $8 \%$ had cough, $4 \%$ had loss of smell, $4 \%$ had loss of taste, $4 \%$ had chest pain (Figure 2) and 40\% of COVID-19 PD patients had fever. Fifteen out of 97 (15\%) COVID-19 HD patients needed ICU admission, 12 out of 97 (12\%) COVID-19 HD patients needed MV. Twenty-two out of 97 (22.6\%) COVID-19 HD patients and 1 out of 5 (20\%) COVID-19 PD patients were on ACEI/ARBs prior to COVID-19 and 1 out of 97 (1\%) COVID-19 HD patients held ACEI/ ARBs. A 33 out of 97 (34\%) COVID-19 HD patients and 4 out of 5 (80\%) COVID-19 PD patients needed to switch to CRRT. Complications including developed myocardial

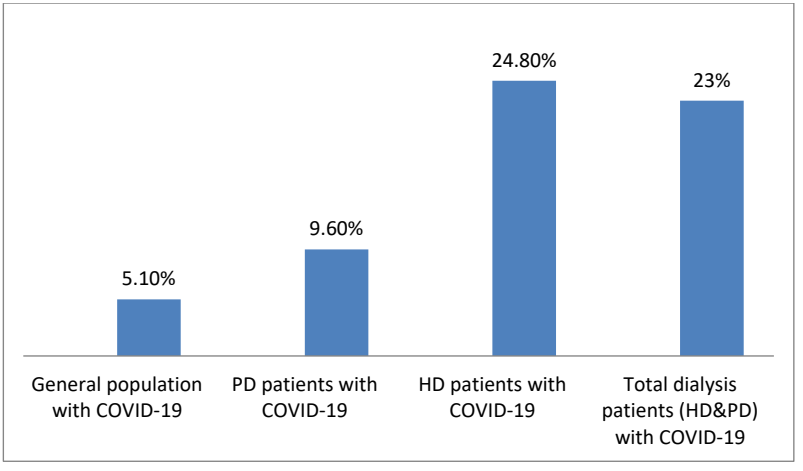

Figure 1. Outbreak of COVID-19 in hemodialysis (HD) and peritoneal dialysis (PD) patients in $\mathrm{Al}$ Khezam dialysis center and general population in Kuwait.

infarction (MI), PE and developed sepsis/acute respiratory distress syndrome (ARDS) were seen in 1 out of $97(1 \%)$ COVID-19 HD patients, 1 out of 97 (1\%) COVID-19 HD patients and 7 out of 97 (7\%) COVID-19 HD patients, respectively. Mortality was 17 (15 $\mathrm{HD}$ and $2 \mathrm{PD}$ ) out of 102 (16.6\%) COVID-19 dialysis patients compared with $0.56 \%$ in general population with COVID-19 infection in Kuwait (Figure 3) and common cause of death was sepsis, MI, heart failure and PE (Table 1).

Table 1. Demographic, clinical characteristics and outcomes of COVID-19 positive dialysis patients

\begin{tabular}{|c|c|c|}
\hline Variables & COVID-19 HD patients $(97 / 390)$ & COVID-19 PD patients $(5 / 52)$ \\
\hline Age (years) & $55.6182 \pm 17.2632$ & $58.5 \pm 9.1$ \\
\hline Gender (M/F) & $46(47 \%) / 51(53 \%)$ & $4(80 \%) / 1(20 \%)$ \\
\hline Nationality (KWT/Non KWT) & $83(86 \%) / 14(14 \%)$ & $3(60 \%) / 2(40 \%)$ \\
\hline DM/HTN/DM+HTN & $61(63 \%) / 89(92 \%) / 62(63 \%)$ & $4(80 \%) / 5(100 \%) / 4(80 \%)$ \\
\hline $\mathrm{IHD} / \mathrm{CHF}$ & $47(48 \%) / 11(11 \%)$ & $3(60 \%) / 0$ \\
\hline Bronchial asthma/COPD & $3(3 \%) / 2(2 \%)$ & $0 / 0$ \\
\hline $\begin{array}{l}\text { Original kidney disease (DN, HTN/GN/Aeropathy/APKD/ } \\
\text { Myeloma/cryo/LMBS/unknown }\end{array}$ & $\begin{array}{l}55(57 \%) / 21(22 \%) / 6(6 \%) / 3(3 \%) / 5(5 \%) / 1(1 \%) / 1 \\
(1 \%) / 1(1 \%) / 4(4 \%)\end{array}$ & $4(80 \%) / 1(20 \%) / 0 / 0 / 0 / 0 / 0 / 0$ \\
\hline $\begin{array}{l}\text { Source of infection (Dialysis center/word/other hospital/ } \\
\text { family member/unknown }\end{array}$ & $8(8 \%) / 2(2 \%) / 3(53 \%) / 38(39 \%) / 46(47 \%)$ & 0/0/0/1 (20\%)/4 (80\%) \\
\hline Fever/fatigue/cough/loss of smell/loss of taste/chest pain & $27(28 \%) / 18(19 \%) / 8(8 \%) / / 4(4 \%) / 4(4 \%) / 4(4 \%)$ & $2(40 \%) / 0 / 0 / 0 / 0 / 0$ \\
\hline ICU/MV/ECMO/Inotropes & $15(15 \%) / 12(12 \%) / / 0 / 2(2 \%)$ & $0 / 0 / 0 / 0$ \\
\hline $\begin{array}{l}\text { Azithromax/clostin/meronem/vancomycin/meropenem/ } \\
\text { tazocin/teicoplanin/klacid }\end{array}$ & $\begin{array}{l}18(18.5 \%) / 20(21 \%) / 18(18.5 \%) / 12(12 \%) / 16 \\
(16 \%) / 10(10 \%) / 2(2 \%) / 2(2 \%)\end{array}$ & $\begin{array}{l}4(80 \%) / 1(20 \%) / 4(80 \%) / 2 \\
(40 \%) / 0 / 0 / 0 / 0\end{array}$ \\
\hline $\mathrm{MI} / / \mathrm{CHF} /$ Arrhythmias/PE & $1(1 \%) / 1(1 \%) / 0 / 1(1 \%)$ & $0 / 0 / 0 / 0$ \\
\hline Sepsis/ARDS & $7(7 \%)$ & 0 \\
\hline ACEI/ARBS prior to COVID-19/Held & $22(22.6 \%) / 1(1 \%)$ & $1(20 \%) / 0$ \\
\hline Need to switch to CRRT & $33(34 \%)$ & $4(80 \%)$ \\
\hline COVID-19 Reinfection & $4(4 \%)$ & 0 \\
\hline Heparin/clexane/fondaparinux/none & $67(69 \%) / 12(12 \%) / 2(2 \%) / 16(16 \%)$ & 0 \\
\hline Mortality & $15(15.4 \%)$ & $2(40 \%)$ \\
\hline With DM/HTN/DM+HTN & $12(80 \%) / 14(93 \%) / 12(80 \%)$ & $2(100 \%) / 2(100 \%) / 2(100 \%)$ \\
\hline With IHD/CHF/IHD+CHF & $11(73 \%) / 3(20 \%) / 1(7 \%)$ & $2(100 \%) / 0 / 0$ \\
\hline Sepsis/MI/PE & $7(47 \%) / / 1(7 \%) / / 2(14 \%)$ & 0/0/1 (100\%) \\
\hline
\end{tabular}

HD, hemodialysis; PD, peritoneal dialysis; DM, diabetes mellitus; HTN, hypertension; IHD, ischemic heart disease; CHF, congestive heart failure; COPD, chronic obstructive pulmonary disease; DN, diabetic nephropathy; GN, glomerulonephritis; APKD, adult polycystic kidney disease; LMBS, Laurance-Moon-BiedleSyndrome; ICU, intensive care unit; MV, mechanical ventilation; ECMO, extra corporeal membrane oxygenation; MI, myocardial infarction; PE, pulmonary embolism; ARDS, acute respiratory distress syndrome, ACEI/ARBS, angiotensin converting enzyme inhibitors/A receptor blockers; CRRT, continuous renal replacement therapy. 


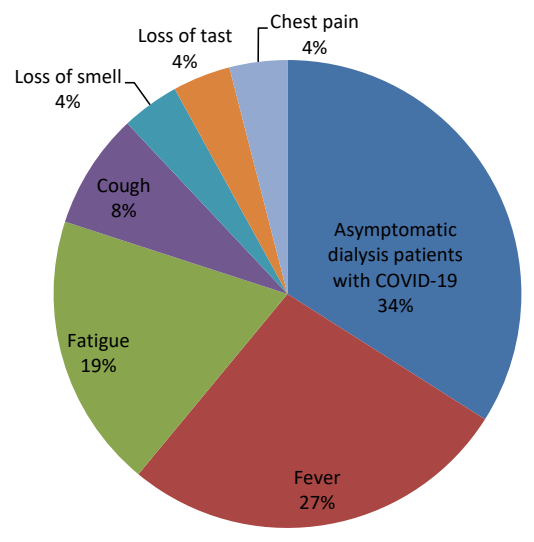

Figure 2. Symptoms of COVID-19 in dialysis patients in Al Khezam dialysis center, Kuwait.

The outbreak of COVID-19 in diabetic patients was comparable with non-diabetic patients (95\% CI -6.5143\% to $42.8959 \%, P=0.1495$ ) while the outbreak of COVID-19 in hypertensive patients was high compared with nonhypertensive patients (95\% CI $32.8048 \%$ to $82.6231 \%$, $P<0.0001)$. Mortality in diabetic and hypertensive patients was high compared with mortality in nondiabetic and non-hypertensive patients (95\% CI 24.3824\% to $78.3175 \%, P=0.0012$; $95 \%$ CI $53.1278 \%$ to $94.0754 \%$, $P<0.0001$ respectively). There was a significant difference in mortality between IHD and non-IHD patients (95\% CI $21.0204 \%$ to $77.3188 \% ; P=0.0026$ ). The presence of DM, HTN and IHD increased mortality, while the presence of, BA and COPD, use or holding of ACEI/ARBS and age did not increase the incidence of COVID-19 or mortality $(P>0.05)$.

\section{Discussion}

In our study, the outbreak of COVID-19 among dialysis patients was $23 \%$ compared with $5.1 \%$ in general population in Kuwait and our study reported that mortality rate among dialysis patients with COVID-19 was $16.6 \%$ compared with $0.56 \%$ in general population with COVID-19 infection in Kuwait. Reinfection was reported in $4 \%$ of COVID-19 HD patients in our study.

Couchoud et al (4) revealed that among dialysis facilities, the COVID-19 prevalence was $6 \%$ during the initial 7-week surge in France as reported from the French Renal Epidemiology and Information Network Registry of patients receiving maintenance dialysis (4). About $3.3 \%$ of patients receiving dialysis nationwide developed COVID-19, with a nearly 2-fold greater risk among patients receiving in-center $\mathrm{HD}$ as compared to patients receiving home dialysis compared with COVID-19 incidence estimates of $0.2 \%$ in the general population (4). In our study, the outbreak of COVID-19 among HD patients $(24.8 \%)$ was high compared with PD patients (9.6\%). Critically, the regional incidence of COVID-19 among patients treated with dialysis mirrored the regional

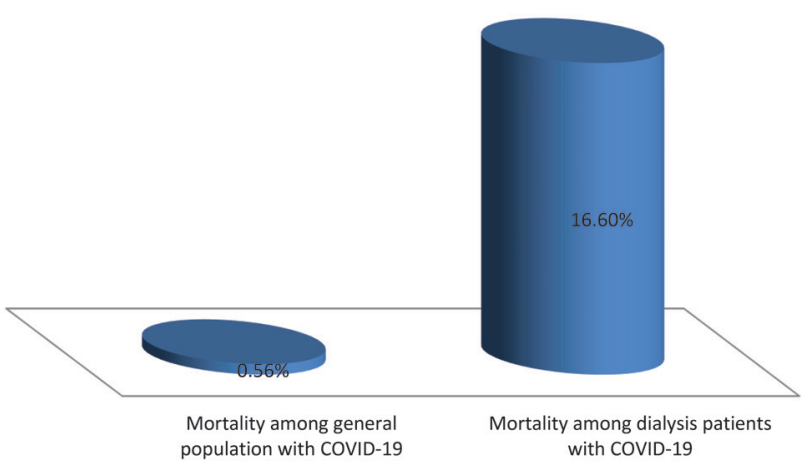

Figure 3. Mortality among dialysis patients with COVID-19 in Al Khezam dialysis center and general population in Kuwait.

incidence in the general population, suggesting a major role in the impact of community spread on infection in patients treated with dialysis.

Among patients receiving dialysis who have COVID-19 mortality was approximately $20 \%$ (4).

This mortality rate is confirmed by Jager et al (5) who, reporting from the European Renal AssociationEuropean Dialysis and Transplant Association Registry, revealed an approximately $20 \%$ mortality rate due to COVID-19 among both patients receiving dialysis and kidney transplant recipients, a rate that is dramatically higher than the estimated $4 \%$ mortality rate overall in Europe among people diagnosed with COVID-19 (6).

Additionally $\mathrm{Ng}$ et al (7) demonstrated the experience of a health system in New York at the height of the first COVID-19 surge, showing that among 419 patients receiving maintenance dialysis who were hospitalized with COVID-19, mortality was $32 \%$, with a $37 \%$ higher adjusted risk of death among patients receiving dialysis who were hospitalized than other patients who were hospitalized with COVID-19. Taken together, these studies revealed the high COVID-19 risk for patients requiring maintenance dialysis, both for acquiring infection and, especially, for poor outcomes once infected, with shortterm mortality that is likely to be $20 \%$ or higher.

In our study, the mortality rate was approximately $16.6 \%$ among patients receiving dialysis in Kuwait. In Europe and much of Asia including Kuwait, aggressive protocols were taken to control the spread of COVID-19 in the general population, with institution of mandatory physical distancing policies in times of high community spread, implementation of mandatory masking policies, and broad availability of contact tracing and testing, including among asymptomatic individuals, with resultant substantial reductions in mortality. In the United States, implementation of and adherence to public health measures have been inconsistent, with the result that the United States still averaged more than 40,000 new cases per day and 750 deaths per day due to COVID-19 in 
September 2020, six months after the initial surge in New York (6).

In centers providing dialysis services, particularly dialysis providers and patients receiving dialysis, must remain vigilant. Interventions to mitigate the impact of COVID-19 among the dialysis population apply to three key settings; the dialysis center, transportation to and from the dialysis center, and the communities in which patients who are treated with dialysis live (8).

Within dialysis facilities, reasonable precautions can decrease the risk of transmission and facilitate care of patients receiving dialysis who have COVID-19, thereby reducing the need for hospitalization and the burden on hospital systems (9).

These include universal mask use among patients and dialysis staff, screening and triage to help identify and rapidly cohort patients with symptoms of or exposure to COVID-19, reduced crowding in HD facilities including in waiting rooms and staff break rooms, specified treatment areas and protocols for providing HD to patients under investigation for or with COVID-19, and, in times of higher community prevalence, testing asymptomatic patients requiring maintenance dialysis as well as dialysis staff for COVID-19. Critically, dialysis facilities should anticipate a continued need for increased physical space and staffing capacity while the pandemic persists, particularly in areas of ongoing community transmission. Patients need education that the easing of physical distancing poses greater risk to them. Transportation to and from dialysis varies greatly around the world; nevertheless, reducing shared rides and mandating sanitation protocols can reduce infection. Lastly, many patients requiring maintenance dialysis reside in high-risk communities, such as densely populated urban areas or congregate residences such as nursing facilities; awareness of the risk posed in these settings and expanded testing of individuals residing in high-risk settings can reduce the threat of COVID-19 both to these patients and to the broader dialysis population $(10,11)$.

\section{Conclusion}

Outbreak and mortality of COVID-19 infection is high in ESKD patients undergoing dialysis compared with general populations. Strict protocol for prevention of COVID-19 should be undertaken in dialysis centers and encourage of home dialysis and highly protective COVID-19 vaccination priority for dialysis patients.

\section{Limitations of the study}

The limitation of the study is that our study is a single center study with few numbers of $\mathrm{HD}$ and $\mathrm{PD}$ patients. We need a big study including all dialysis centers in Kuwait.
Acknowledgements

Many thanks to all my colleagues in Al Khezam dialysis center for following, caring and management and helping in collecting data of dialysis patients infected with COVID-19.

\section{Authors' contribution}

EA was the principal investigator of the study. EA, BA, and RA were included in the preparation of concept and design. EA, GN, SA, and $\mathrm{MH}$ revisited the manuscript and critically evaluated the intellectual contents. All authors participated in preparing the final draft of the manuscript, revised the manuscript and critically evaluated the intellectual contents. All authors have read and approved the contents of the manuscript and confirmed the accuracy or the integrity of any part of the work.

\section{Conflicts of interests}

The authors declare that they have no competing interests.

\section{Ethical considerations}

Ethical issues (including plagiarism, data fabrication, double publication) have been completely observed by the authors.

\section{Funding/Support}

The authors received no financial support for the research, authorship, and/or publication of this article

\section{References}

1. Pisani A, Rizzo M, AngelucciV, Riccio E. COVID-19 experience in hemodialysis patients: a cue for therapeutic heparin-based strategies? Nephron. 2020;144:383-5.

2. Ikizler TA. COVID-19 and dialysis units: what do we know now and what should we do? Am J Kidney Dis. 2020;76:1-3. doi: 10.1053/j.ajkd.2020.03.008.

3. Yun Tang, BS, Yi Li, PhD, Yi Xin, BS, Fei Deng, MBBS. Prevention and Management of COVID-19 in Hemodialysis Centers. Am J Manag Care. 2020;26:e237-8.

4. Couchoud C, Bayer F, Ayav C. Low incidence of SARS-CoV-2, risk factors of mortality and the course of illness in the French national cohort of dialysis patients. Kidney Int. 2020;98:151929. doi: 10.1016/j.kint.2020.07.042.

5. Jager KJ, Kramer A, Chesnaye NC. Results from the ERA-EDTA Registry indicate a high mortality due to COVID-19 in dialysis patients and kidney transplant recipients across Europe. Kidney Int. 2020;98:1540-8. doi: 10.1016/j.kint.2020.09.006

6. World Health Organization. WHO coronavirus disease (COVID-19) dashboard. 2020. https://covid19.who.int/. Accessed October 2, 2020.

7. $\mathrm{Ng} \mathrm{JH}$, Hirsch JS, Wanchoo R. Outcomes of patients with endstage kidney disease hospitalized with COVID-19. Kidney Int. 2020;98:1530-9. doi: 10.1016/j.kint.2020.07.030.

8. Weiner D.E. Watnick S.G. Hemodialysis and COVID-19: an Achilles' heel in the pandemic health care response in the United States. Kidney Med. 2020;2:227-30.

9. Centers for Disease Control and Prevention. Interim additional guidance for infection prevention and control recommendations for patients with suspected or confirmed COVID-19 in outpatient hemodialysis facilities. https://www. cdc.gov/coronavirus/2019-ncov/hcp/dialysis.html. 2020.

10. Bhayani S, Sengupta R, Markossian T. Dialysis, COVID-19, poverty, and race in greater Chicago: an ecological analysis. Kidney Med. 2020;2:552-558.

11. Liu CK, Ghai S, Waikar SS, Weiner DE. COVID-19 infection risk among hemodialysis patients in long-term care facilities. Kidney Med. 220;2:810-1. doi: 10.1016/j.xkme.2020.07.005. 